\title{
Failure Mechanisms of Alloys with a Bimodal Graine Size Distribution
}

\author{
Vladimir A. Skripnyak, Evgeniya G. Skripnyak, and Vladimir V. Skripnyak
}

\begin{abstract}
A multi-scale computational approach was used for the investigation of a high strain rate deformation and fracture of magnesium and titanium alloys with a bimodal distribution of grain sizes under dynamic loading. The processes of inelastic deformation and damage of titanium alloys were investigated at the mesoscale level by the numerical simulation method. It was shown that localization of plastic deformation under tension at high strain rates depends on grain size distribution. The critical fracture stress of alloys depends on relative volumes of coarse grains in representative volume. Microcracks nucleation at quasi-static and dynamic loading is associated with strain localization in ultra-fine grained partial volumes. Microcracks arise in the vicinity of coarse and ultrafine grains boundaries. It is revealed that the occurrence of a bimodal grain size distributions causes increased ductility, but decreased tensile strength of UFG alloys. The increase in fine precipitation concentration results not only strengthening but also an increase in ductility of UFG alloys with bimodal grain size distribution.
\end{abstract}

Keywords Bimodal distribution of grain sizes $\cdot$ Computational plasticity $\cdot$ High strain rates $\cdot$ Titanium alloys $\cdot$ Magnesium alloys

\section{Introduction}

Hexagonal close-packed (HCP) metals, such as $\mathrm{Ti}, \mathrm{Mg}, \mathrm{Zn}$ and $\mathrm{Zr}$, are of interest for engineering and medical applications due to their unique combination of high ductility and strength. In recent years, intensive efforts have been focused on investigations of the physical and mechanical properties of alloys, whose grain structure is formed by surface rolling, severe plastic deformation, selective laser melting, or selective laser sintering, and friction stir processing [1-5].

Recently Long et al. showed a bimodal microstructure formation in the ultrafinegrained Ti-6Al-4V alloy produced by means of the spark plasma sintering of

V. A. Skripnyak (凶) · E. G. Skripnyak · V. V. Skripnyak

National Research Tomsk State University (TSU), 36 Lenin Avenue, 634050 Tomsk, Russia

e-mail: skrp2006@yandex.ru 
a mixture of ball-milled and unmilled powders [6]. The additive manufacturing technology of HCP metals opens new possibilities for obtaining parts from alloys combining high strength, ductility, and fracture toughness.

Usually, the formation of nano-sized and ultra-fine grained structures in metal alloys leads to increased strength and decreased ductility. The low ductility of ultrafine (UFG) alloys limits their applications as advanced engineering materials. In recent years it was found that the alloys with a bimodal grain size distribution may exhibit increased yield strength without reducing ductility [7, 8]. Guo showed the ductility of fine-grained (FG) $\mathrm{Zr}$ with an average grain size of $2-3 \mu \mathrm{m}$ was $17 \%$ higher than that of coarse-grained alloy [2]. Pozdnyakov [9], Malygin [10], Ulacia [11] showed that the bimodal grain distribution can be formed in light alloys by means of severe plastic deformation and follow-up heat treatment. It was revealed that hexagonal close-packed (HCP) alloys with a bimodal grain size distribution exhibit a number of anomalies in mechanical behavior $[12,13]$. Therefore, the optimization of strength and ductility requires a profound knowledge of volume fraction and distribution of ultra-fine grains and coarse grain in multi-modal alloys. Research of the influence of the grain structure on the strength and ductility of the alloys under cyclic loadings, dynamic loadings, quasistatic loadings are carried out using experimental methods and by computer simulation [14-17].

Berbenni proposed a theoretical micromechanical model taking into account the grain size distribution and representing the accommodation of grain deformation in HCP metals (Zirconium- $\alpha$ ) [7]. Raeisinia proposed a model to examine the effect of unimodal and bimodal grain size distributions on the uniaxial tensile behavior of a number of polycrystals [12]. The model demonstrated that bimodal grain size distributions have enhanced macro mechanical properties as compared with their unimodal counterparts. The authors of this article proposed a multiscale model of UFG metals with bimodal grain size to predict the localization of plastic flow in UFG light alloys under dynamic loads with respect to the ratio of volume concentrations of small and large grains. Results of calculations have shown increased dynamic ductility of UFG titanium and magnesium alloys, when the specific volume of coarse grains is greater than $30 \%$.

Zhu proposed a micromechanics-based model to investigate the mechanical behavior of polycrystalline dual-phase metals with a bimodal grain size distribution, and fracture by means of nano/microcracks generation during plastic deformation. Results have shown that the volume fraction of coarse grains controls the strength and ductility of metals [18-21]. Clayton developed a continuum model of crystal plasticity for the interpretation of experimental data on shock wave propagation and spall fracture in polycrystalline aggregates [22]. The model accounted for complex features of the mechanical response of alloys: plastic anisotropy, large volumetric strain, heat conduction, thermos-elastic heating, rate- and temperaturedependent flow stress. Magee proposed a multiscale modeling technique to simulate the microstructural deformation of an alloy with bimodal grain size distribution. The simulation has shown that intergranular cracks nucleate at the coarse grains/ultrafined grains interfaces [23]. Although much research is carried out on the mechanical properties of alloys with a bimodal grain size distribution, a number of issues remains 
poorly understood. The effect of the bimodal grain structure of alloys on the ultimate strain to failure at high strain rates is poorly studied [24]. The laws of localization of plastic deformation and the laws of damage accumulation in HCP alloys with a bimodal grain size distribution under dynamic impacts are not well studied. These problems are of great practical importance and are associated with the transition to new technologies for digital design and production of technical and medical products.

In this paper, we develop a multilevel approach to study the mechanical behavior of HCP alloys in a wide range of strain rates.

\section{Computational Model}

Titanium, zirconium, and magnesium alloys with a hexagonal closed-packed (HCP) structure have a significant low crystal symmetry compared to the face-centered cubic (FCC) and body-centered cubic (BCC) crystal structures of engineering alloys. The mechanical behavior of HCP alloys under quasistatic and dynamic loading at temperatures $T / T_{m}$ less than 0.5 is determined by dislocation mechanisms and twinning [25-29]. Authors used modifications of the constitutive equations developed in the framework of the micro-dynamical approach and taking into account the thermally activated dislocation mechanisms [15-17, 30-32].

The grain sizes and the grain-boundary phase structure affect the glide of dislocations and the formation of dislocation substructures during the plastic flow [27].

Mechanical response of polycrystalline alloys can be described by parameters of states averaging over the model representative volume element (RVE). Therefore, it is required for model volume to represent not only a given grain size distribution but also realistic values of the physical properties of the system.

We use the approach of a finite mixture model for the estimation of average sizes in multimodal grain size distribution [7, 17, 33].

The multimodal grain size distribution described by probability density function $g\left(d_{g}\right)$. Distribution $g\left(d_{g}\right)$ is a mixture of $k$ component distributions $g_{1}, g_{2}, g_{3}$ of ultra-fined grains (UFG), fine grains (FG), coarse grains (CG), respectively:

$$
g\left(d_{g}\right)=\sum_{k=1}^{m} \lambda_{k} g_{k}\left(d_{g}\right),
$$

where $\lambda_{k}$ are the mixing weights, $\lambda_{k}>0, \sum_{k=1}^{3} \lambda_{k}=1, k=1,2,3$.

This method allows us to determine the multimodal grain size distribution function and the range of grain size groups (UFG, FG, CG). In this paper, we present simulation results for unimodal and bimodal (UFG and CG) grain distribution. We used experimental data of grain size distribution of alloys after severe plastic deformation to calibrate the computational model. 
The RVE can be created using the experimental data on grain structures obtained by the analysis of Electron Backscatter Diffraction (EBSD) based on scanning electron microscopy (SEM) [34-36]. This analysis gives quantitative information about sizes and shapes of grains, Euler angles, angles of misorientation at the grain/subgrain boundaries with angular resolution $\sim 0.5^{\circ}$. The shape coefficient was determined by the relation of minimal grain size to maximal size $\left(\xi_{k}=d_{g}^{\min } / d_{g}^{\max }\right)$, where $k$ is the number of grain size group.

The analysis of grain structure distributions has shown that there are several types of grains structure characterized by unimodal (near log-normal) distribution, and bimodal grain size distribution, multimodal grain size distribution [2, 7, 8, 35].

The specific volume of ultrafine grains (with grain size $100 \mathrm{~nm}<$ $\left.d_{g}<1 \mu \mathrm{m}\right)$, microstructural grains $\left(1<d_{g}<10 \mu \mathrm{m}\right)$, and coarse grains $\left(10 \mu \mathrm{m}<d_{g}<d_{g} \max \right)$ was estimated using the probability density function $g\left(d_{g}\right)$ of grain size distribution:

$$
C_{U F G}=\int_{d_{g}^{\min }}^{1 \mu \mathrm{m}} g_{1}(x) d x, \quad C_{F G}=\int_{1 \mu \mathrm{m}}^{10 \mu \mathrm{m}} g_{2}(x) d x, \quad C_{C G}=\int_{10 \mu \mathrm{m}}^{d_{g}^{\max }} g_{3}(x) d x,
$$

where $C_{U F G}, C_{F G}, C_{C G}$ are specific volumes of UFG, FG, and CG, $g_{1}, g_{2}, g_{3}$ are the probability density functions of UFG, FG, and CG systems, respectively.

The relative volume of coarse and ultra-fine grains in model RVE was determined in accordance with the probability density function of the grain size distribution.

Figure 1 shows a 3D RVE model of a titanium alloy with a bimodal grain size distribution. An RVE with dimensions of $14 \times 8 \times 1(\mu \mathrm{m})^{3}$ was used.

Computational domains were meshed with eight-node linear bricks and reduced integration together with hourglass control.

The kinematic boundary conditions correspond to macroscopic tension. The scheme of boundary conditions is shown in Fig. 1.

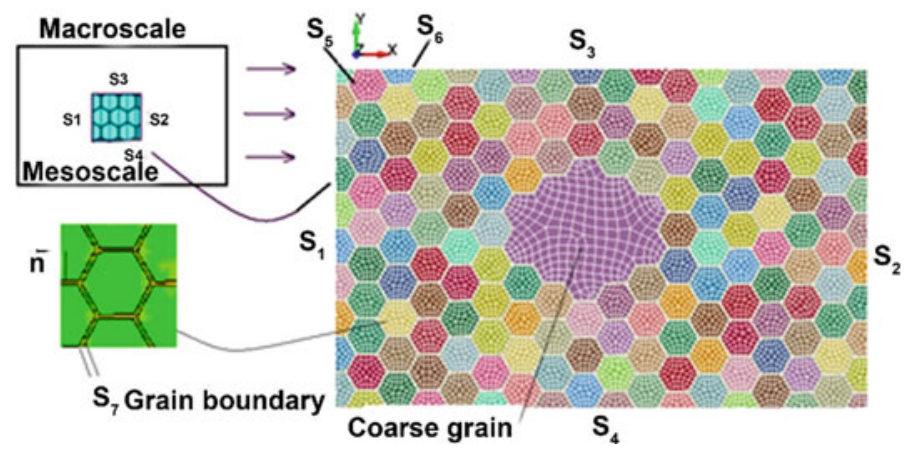

Fig. 1 Scheme of boundary conditions 


$$
\begin{aligned}
& u_{x}\left(x_{k}, t\right)=0, \quad x_{k} \in S_{1} \\
& u_{x}\left(x_{k}, t\right)=v_{x}, \quad x_{k} \in S_{2} \\
& \sigma_{22}=0, \quad x_{k} \in S_{3}, \quad x_{k} \in S_{4} \\
& u_{z}=0, \quad x_{k} \in S_{5}, \quad x_{k} \in S_{6} \\
& u_{k}^{A}-u_{k}^{B}=0, \quad \sigma_{n}^{A}=-\sigma_{n}^{B}, \quad k=1,2,3, \quad x_{k} \in S_{7}
\end{aligned}
$$

where $u_{k}^{A}, u_{k}^{B}$ are the projections of the displacement rate onto the external normal to the boundary $S_{7}$ of the grain and the grain boundary phase at the boundary points, respectively, $\sigma_{n}^{A}, \sigma_{n}^{B}$ are the components of the surface forces along the external normal $\vec{n}$ to the boundary $S_{7}$, respectively, $x_{k}$ is the Cartesian coordinate.

Mechanical behavior at the mesoscale level is described within the approach of the damaged elastic-plastic medium. The system of equations includes:

Conservation equations (4);

Kinematic relations (5);

Constitutive relations (8);

Equation of State (9);

Relaxation equation for the deviatoric stress tensor (10).

$$
\frac{d \rho}{d t}=\rho \frac{\partial u_{i}}{\partial x_{i}}, \quad \frac{\partial \sigma_{i j}}{\partial x_{j}}=\rho \frac{d u_{i}}{d t}, \quad \rho \frac{d E}{d t}=\sigma_{i j} \dot{\varepsilon}_{i j},
$$

where $\rho$ is the mass density, $u_{i}$ is the components of the particle velocity vector, $x_{i}$ is the Cartesian coordinates, $i=1,2,3, E$ is the specific internal energy, $\sigma_{i j}$ are the components of the effective stress tensor of the damaged medium, $\dot{\varepsilon}_{i j}$ are the components of strain rate tensor.

Kinematics of the medium was described by the local strain rate tensor:

$$
\left.\left.\dot{\varepsilon}_{i j}=(1 / 2)\left[\partial u_{i} / \partial x_{j}+\partial u_{j} / \partial x_{i}\right)\right], \quad \dot{\omega}_{i j}=(1 / 2)\left[\partial u_{i} / \partial x_{j}-\partial u_{j} / \partial x_{i}\right)\right],
$$

where $\dot{\varepsilon}_{i j}, \dot{\omega}_{i j}$ are the components of strain rate tensor and the bending-torsion tensor, $u_{i}$ is the component of particles velocity vector.

The components of strain rate tensor are expressed by the sum of elastic and inelastic terms:

$$
\dot{\varepsilon}_{i j}=\dot{\varepsilon}_{i j}^{e}+\dot{\varepsilon}_{i j}^{p}, \quad \dot{\varepsilon}_{i j}^{p}=\dot{e}_{i j}^{p}+\delta_{i j} \dot{\varepsilon}_{k k}^{p} / 3,
$$

where $\dot{\varepsilon}_{i j}^{e}$ are the components of the elastic strain rate tensor, $\dot{\varepsilon}_{i j}^{p}$ are the components of the inelastic strain rate tensor.

The bulk inelastic strain rate is described by relation:

$$
\dot{\varepsilon}_{k k}^{p}=\dot{f}_{\text {growth }} /(1-f),
$$


where $f$ is the damage parameter, the substantial time derivative is denoted via dot notation.

The bulk inelastic strain rate $\varepsilon_{k k}^{p}$ is equal to zero only when the material is undamaged.

$$
\begin{gathered}
\sigma_{i j}=\sigma_{i j}^{(m)} \varphi(f), \quad \sigma_{i j}^{(m)}=-p^{(m)} \delta_{i j}+S_{i j}^{(m)} \\
p^{(m)}=p_{x}^{(m)}(\rho)+\Gamma(\rho) \rho E_{T}, \quad E_{T}=C_{p} T \\
p_{x}^{(m)}=\frac{3}{2} B_{0} \cdot\left(\left(\rho_{0} / \rho\right)^{-7 / 3}-\left(\rho_{0} / \rho\right)^{-5 / 3}\right)\left[1-\frac{3}{4}\left(4-B_{1}\right) \cdot\left(\left(\rho_{0} / \rho\right)^{-2 / 3}-1\right)\right] \\
D S_{i j}^{(m)} / D t=2 \mu\left(\dot{\varepsilon}_{i j}^{e}-\delta_{i j} \dot{\varepsilon}_{k k}^{e} / 3\right), \quad \dot{e}_{i j}^{p}=\lambda \partial \Phi / \partial \sigma_{i j},
\end{gathered}
$$

the function $\varphi(f)$ establishes a relation between the effective stresses of the damaged medium and the stresses in the condensed phase, $\Gamma$ is the Grüneisen coefficient, $\rho_{0}$ is the initial mass density of the condensed phase of the alloy, $\gamma_{R}, \rho_{R}, n, B_{0}, B_{1}$ are the material's constants, $C_{p}$ is the specific heat capacity, $D(\cdot) / D t$ is the Jaumann derivative, $\mu$ is the shear modulus, $\dot{f}_{\text {growth }}$ is the void growth rate, $f$ is the void volume fraction in the damaged medium, $\dot{\lambda}$ is the plastic multiplier derived from the consistency condition $\dot{\Phi}=0$, and $\Phi$ is the plastic potential. The plastic potential was described using the Gurson-Tvergaard model (GTN) [32, 37-39].

The Grüneisen coefficient $\Gamma$ was equal to 1.42 and 1.09 for $\mathrm{Mg}-3 \mathrm{Al}-1 \mathrm{Zn}$ and Ti-5Al-2.5Sn, respectively.

The function $\varphi(f)$ takes the form of $(1-f)$ for pressure and is implicitly defined for the deviatoric stress tensor [40].

The temperature rise associated with energy dissipation during plastic flow can be evaluated by relation $[25,32]$ :

$$
T=T_{0}+\int_{0}^{\varepsilon_{e q}^{p}}\left(\beta / \rho C_{p}\right) \sigma_{e q} d \varepsilon_{e q}^{p},
$$

where $T_{0}$ is the initial temperature and $\beta \sim 0.9$ is the parameter representing a fraction of plastic work converted into heat.

The specific heat capacity for Ti-5Al-2.5Sn titanium was calculated by the phenomenological relations within the temperature range 293-1115 K [32]:

$$
C_{p}=248.389+1.53067 T-0.00245 T^{2}(\mathrm{~J} / \mathrm{kg} \mathrm{K}) \text { for } 0<T<T_{\alpha \beta}=1320 \mathrm{~K},
$$

The temperature dependence of the shear modulus for alpha titanium alloy was described by the equation: 


$$
\mu(T)=48.66-0.03223 T(\mathrm{GPa}), \quad(273 K<T<1200 \mathrm{~K}) .
$$

The flow stress was described by equation:

$$
\begin{aligned}
\sigma_{s}= & \sigma_{s 0} \exp \left\{C_{1} \sqrt{\left(1-T / T_{m}\right)}\right\}+C_{2} \sqrt{1-\exp \left\{-k_{0} \varepsilon_{e q}^{p}\right\}} \\
& \exp \left\{-C_{3} T\right\} \exp \left\{C_{4} T \ln \left(\dot{\varepsilon}_{e q} / \dot{\varepsilon}_{e q 0}\right)\right\},
\end{aligned}
$$

where $\dot{\varepsilon}_{e q}=\left[(2 / 3) \dot{\varepsilon}_{i j} \dot{\varepsilon}_{i j}\right]^{1 / 2}, \dot{\varepsilon}_{e q 0}=\gamma_{1} \exp \left\{-T / \gamma_{2}\right\}+\gamma_{3}, \varepsilon_{e q}^{p}=\int_{0}^{t} \dot{\varepsilon}_{e q}^{p} d t$ is the equivalent plastic strain $\gamma_{1}=2115.08615 \mathrm{~s}^{-1}, \gamma_{2}=38.26589 \mathrm{~K}, \gamma_{3}=9.82388 \times$ $10^{-5} \mathrm{~s}^{-1}$ and $T_{m}$ is the melting temperature, $\dot{\varepsilon}_{e q 0}=1.0 \mathrm{~s}^{-1}$.

$$
\sigma_{s}=\sigma_{s 0}+C_{5}\left(\varepsilon_{e q}^{p}\right)^{n_{1}}+k_{h p} d_{g}^{-1 / 2}-C_{2} \exp \left\{-C_{3} T+C_{4} T \ln \left(\dot{\varepsilon}_{e q} / \dot{\varepsilon}_{e q 0}\right)\right\}
$$

where $\sigma_{s 0}, C_{5}, n_{1}, k_{h p}, C_{2}, C_{3}, C_{4}$ are the material parameters, $d_{g}$ is the average grain size.

The impact of grain size distribution on the stress of hep alloys with average grain sizes in single-mode and bimodal microstructures was taken into account in Eq. (15) by analogy with the Hall-Petch relation.

Material parameters of Ti-5Al-2.5Sn and $\mathrm{Mg}-3 \mathrm{Al}-1 \mathrm{Zn}$ are shown in Tables 1 and 2 , respectively.

The influence of damage on the flow stress was taken into account using the Gurson-Tvergaard model [32, 36, 38]:

$$
\left(\sigma_{e q}^{2} / \sigma_{s}^{2}\right)+2 q_{1} f^{*} \cosh \left(-q_{2} p / 2 \sigma_{s}\right)-1-q_{3}\left(f^{*}\right)^{2}=0,
$$

where $\sigma_{s}$ is the yield stress and $q_{1}, q_{2}$ and $q_{3}$ are the model parameters, $\sigma_{e q}=$ $\sqrt{\frac{3}{2} \sigma_{i j} \sigma_{i j}-\frac{1}{2} \sigma_{k k} \sigma_{k k}}$.

$$
\begin{aligned}
\dot{f} & =\dot{f}_{\text {nucl }}+\dot{f}_{\text {growth }}, \\
\dot{f}_{\text {nucl }} & =\varepsilon_{e q}^{p}\left(f_{N} / s_{N}\right) \exp \left\{-0.5\left[\left(\varepsilon_{e q}^{p}-\varepsilon_{N}\right) / s_{N}\right]^{2}\right\}, \\
\dot{f}_{\text {growth }} & =(1-f) \dot{\varepsilon}_{k k}^{p},
\end{aligned}
$$

Table 1 Material parameters of Eq. (14)

\begin{tabular}{l|l|l|l|l|l|l|l}
\hline Parameter & $\sigma_{s 0}(\mathrm{GPa})$ & $C_{1}$ & $C_{2}(\mathrm{GPa})$ & $C_{3}\left(\mathrm{~K}^{-1}\right)$ & $C_{4}\left(\mathrm{~K}^{-1}\right)$ & $k_{0}$ & $T_{m}(\mathrm{~K})$ \\
\hline Ti-5Al-2.5Sn & 0.02 & 3.85 & 0.56 & 0.0016 & 0.00009 & 8.5 & 1875 \\
\hline
\end{tabular}

Table 2 Material parameters of Eq. (15)

\begin{tabular}{l|l|l|l|l|l|l|l}
\hline Coefficients & $\sigma_{s 0}(\mathrm{GPa})$ & $k_{h p}\left(\mathrm{~Pa} \mathrm{~nm}^{1 / 2}\right)$ & $C_{2}(\mathrm{GPa})$ & $C_{3}\left(\mathrm{~K}^{-1}\right)$ & $C_{4}\left(\mathrm{~K}^{-1}\right)$ & $C_{5}(\mathrm{GPa})$ & $n_{1}$ \\
\hline $\mathrm{Mg}-3 \mathrm{Al}-1 \mathrm{Zn}$ & 0.141 & 6.2 & 0.315 & 0.0029 & 0.000389 & 0.505 & 0.2514 \\
\hline
\end{tabular}


Table 3 Dimensionless parameters for the Gurson-Tvergaard-Needleman (GTN) model for alpha titanium and magnesium alloys

\begin{tabular}{l|l|l|l|l|l|l|l|l|l}
\hline & $q_{1}$ & $q_{2}$ & $q_{3}$ & $f_{0}$ & $f_{N}$ & $f_{C}$ & $f_{f}$ & $\varepsilon_{N}$ & $S_{N}$ \\
\hline Ti-5Al-2.5Sn & 1.3 & 1 & 1.69 & 0.00 & 0.2 & 0.035 & 0.4 & 0.28 & 0.1 \\
\hline $\mathrm{Mg}-3 \mathrm{Al}-1 \mathrm{Zn}$ & 1.5 & 1 & 2.25 & 0.00 & 0.4 & 0.073 & 0.128 & 0.1 & 0.2 \\
\hline
\end{tabular}

where $\varepsilon_{N}$ and $S_{N}$ are the average nucleation strain and the standard deviation, respectively. The amount of nucleating voids is controlled by the parameter $f_{N}$

$$
\begin{aligned}
& f^{*}=f \text { for } f \leq f_{c} \\
& f^{*}=f_{c}+\left(\bar{f}_{F}-f_{c}\right) /\left(f_{F}-f_{c}\right) \text { for } f>f_{c},
\end{aligned}
$$

where $\bar{f}_{F}=\left(q_{1}+\sqrt{q_{1}^{2}-q_{3}}\right) / q_{3}$.

The final stage in ductile fracture comprises the voids coalescence [38]. This causes softening of the material and accelerated growth rate of the void fraction $f^{*}$.

The model parameters for Ti-5 Al-2.5Sn and $\mathrm{Mg}-3 \mathrm{Al}-1 \mathrm{Zn}$ were determined using numerical simulation. These parameters are given in Table 3.

We use the ductile fracture criteria for alloys at the room and elevated temperatures owing to relatively low melting temperature [31, 38, 41].

Finite elements are removed from the grid model and free boundary conditions are introduced at the formed boundary, when the local fracture criterion is met.

Specific features of mechanical properties of nanostructured materials are connected to distinctions of matter properties in a crystalline phase of grains and in the boundary of grains. A decrease in the mass density of nanostructured materials is caused by increased defect's density and relative volume of grain boundary phase.

\section{Results and Discussion}

Figure 2 shows the fields of equivalent plastic strain under tension at $v_{x}=2.3 \mathrm{~m} / \mathrm{s}$. Damages were localized near the grain boundary of coarse grains.

Damage nucleation in alloys with a bimodal grain size distribution occurs in shear bands and zones of their intersection.

These results agree with experimental observations of strain localization and fracture of titanium alloys by Sharkeev [42], authors of this work [32, 43], Valoppi [44] and Zheng [45]. It is significant that increases in fine precipitates concentration in alloys caused the increase in resistance to plastic flow within both coarse and ultrafine grains. 

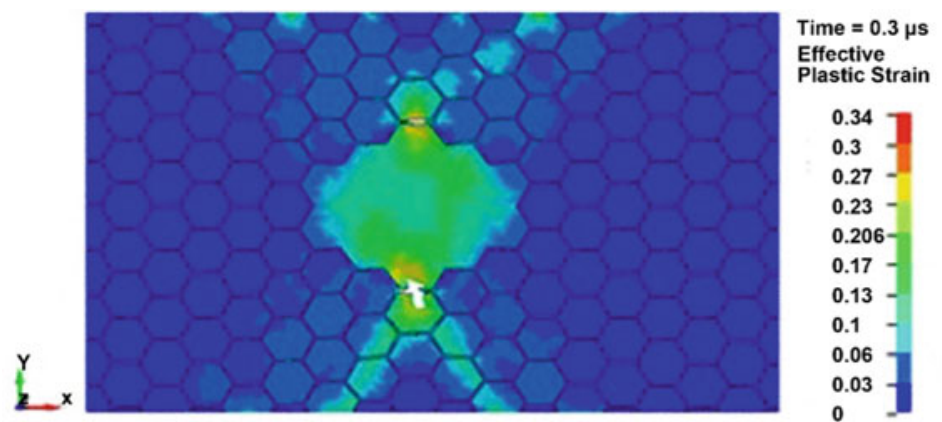

(a)
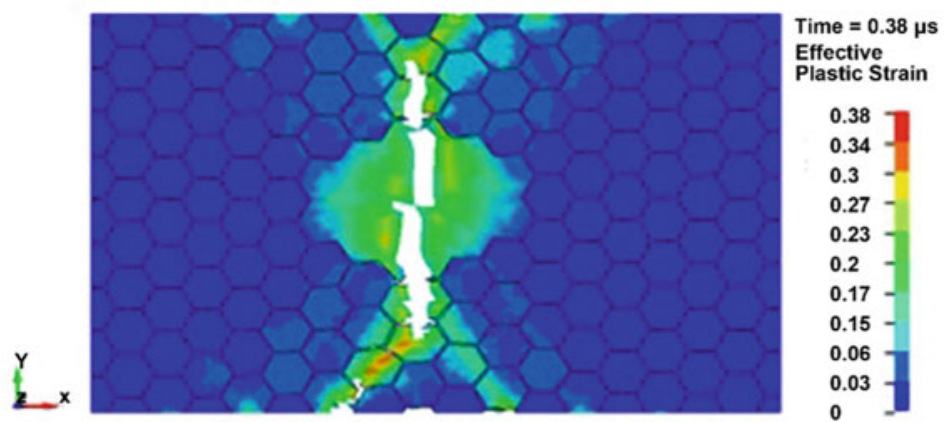

(b)
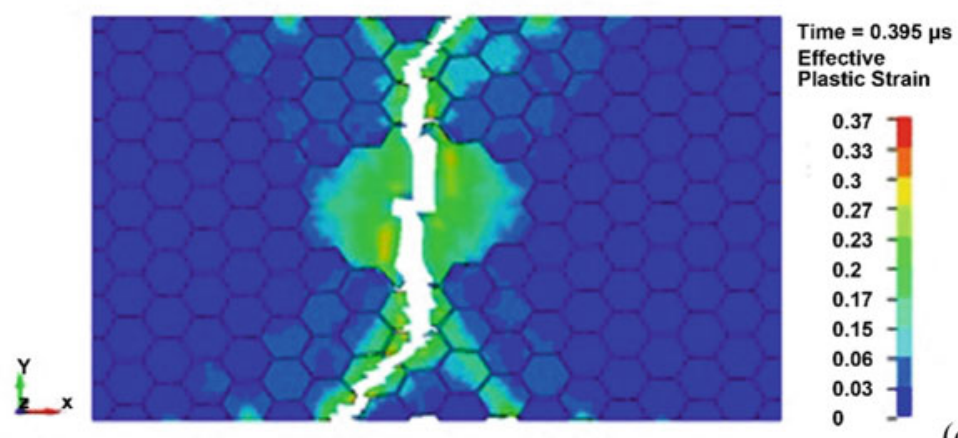

(c)

Fig. 2 Effective plastic strain field at time, a $0.332 \mu$ s, b $0.3895 \mu$ s, c $0.398 \mu \mathrm{s}$ in Ti-5Al-2.5Sn alloy with bimodal grain structure

Thus, the fracture of alloys with bimodal grain structures is caused by the damage nucleation at the boundary of coarse grains with an ultrafine-grained structure and further growth of damage in mesoscopic bands of localized plastic deformation.

Thus, the process of fracture of alloys with bimodal grain structures is associated with the formation of mesoscopic bands of localized plastic deformation.

The segregation of impurity atoms in the grain-boundary phase affects the formation of plastic shear bands [14, 46]. 
The mechanical properties of the grain boundary phase were varied to take into account the effect of segregation of impurity atoms on the yield stress in the simulation.

Figure $2 b$ shows the field of equivalent strains, indicating that the formation of cracks in large grains can be accompanied by a change in the orientation of the shear localization bands at the mesoscopic level. This occurs as a result of a change in the orientation of the plane of maximum shear stresses during the evolution of a triaxial stress state near cracks. A macroscopic crack obtained by modeling the tension of flat samples has the same configuration as the cracks observed in experimental studies by Verleysen [24] and authors of this work [31, 32].

The averaged strain along the axis of tension of the computational domain at the moment of crack crossed the representative volume was interpreted as the ultimate strain to fracture of the alloy.

Figure 3 shows the dependence of strain to fracture of titanium and magnesium alloys on the logarithm of strain rates. Experimental data reported by Ulacia for coarse-grained $\mathrm{Mg}-3 \mathrm{Al}-1 \mathrm{Zn}$ alloy are marked by filled square symbols [11]. The experimental data for the Ti-5Al-2.5Sn alloy are shown by filled triangular symbols $[32,47]$.

Strains to fracture behave nonmonotonically and nonlinearly with increasing strain rate in the range from 0.001 to 1000 1/s as shown in Fig. 3 (Curves 1 and 3). In coarse-grained HCP alloys, this is related to more intense twinning under dynamic loading.

Curve 2 is obtained by the approximation of calculated values of the strains to fracture under tension of a magnesium alloy with a bimodal grain structure with a specific volume of large grains of $70 \%$. With an increase in the concentration of micron and

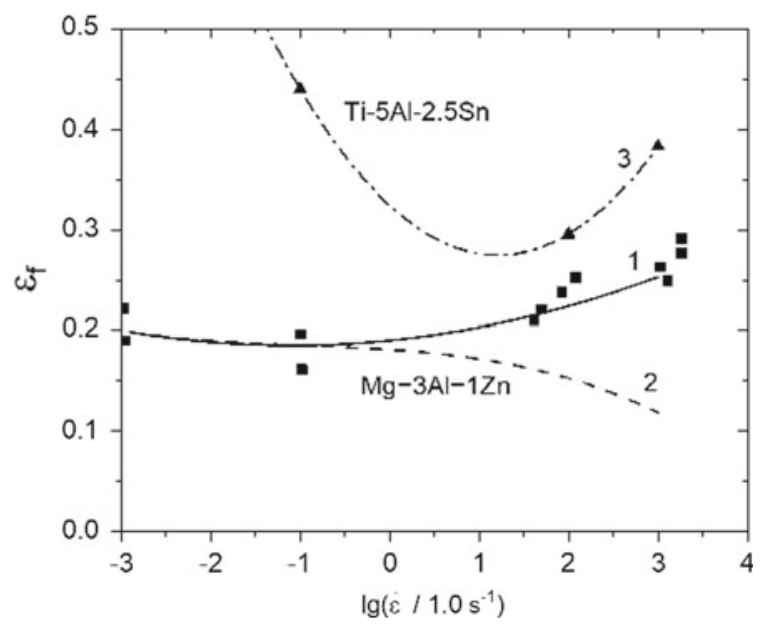

Fig. 3 The strain to fracture versus logarithm of strain rates for magnesium and titanium alloys with a unimodal (curve 2,3) and a bimodal grain sizes distribution (curve 1, the concentration of coarse grains is $70 \%$ ) 
submicron grains in the alloy, the strain to failure under tension decreases nonlinearly. The simulation results agree with the available experimental data [11, 45-50].

The strain to fracture of alloy with bimodal grain size distribution versus specific volume of coarse grains under quasi-static tension can be described by the relation:

$$
\varepsilon_{f}^{n}=0.01 \exp \left(C_{c g} / 0.363\right)
$$

where $\varepsilon_{f}^{n}$ is the strain to fracture under quasi-static tension, $C_{c g}$ is the specific volume of coarse grains.

Equation (19) describes the ductility of alloys with a bimodal grains distribution versus the specific volume of coarse grains. The increase in ductility of HCP alloys under quasi-static tension occurs when a specific volume of coarse grains is greater than $30 \%$.

\section{Conclusions}

The multiscale approach was used in the computer simulation of fracture of magnesium and titanium alloys at high strain rates. Structured RVEs were proposed to predict the mechanical properties of alloys taking into account the grain size distribution and the segregation impurity atoms in the grain-boundary.

The results of computer simulation showed that damage nucleation in alloys with a bimodal grain size distribution occurs in the shear bands and their intersection zones. Damage arises at the boundary between coarse- and ultrafine-grained structures. Further damage growth occurs in the mesoscopic bands of localized plastic deformation.

Thus the computer simulation can be used to estimate the influence of grains size distribution on the dynamic strength and ductility of HCP alloys.

Localization of plastic flow in HCP alloys with bimodal grain size distribution under tension at high strain rates depends on the ratio between volume concentrations of fine and coarse grains. As a result, the strain to fracture of hcp alloys with bimodal grain size distribution varies nonlinearly with tensile strain rate in the range from 0.001 to $10001 / \mathrm{s}$.

The dynamic ductility of HCP alloys with bimodal grain size distribution is increased when a specific volume of coarse grains is greater than $30 \%$.

\section{References}

1. Fall A, Monajati H, Khodabandeh A, Fesharaki MH, Champliaud H, Jahazi M (2019) Local mechanical properties, microstructure, and microtexture in friction stir welded Ti-6Al-4V alloy. Mater Sci Eng: A 749:166-175. https://doi.org/10.1016/j.msea.2019.01.077 
2. Guo D, Zhang Z, Zhang G, Li M, Shi Y, Ma T, Zhang X (2014) An extraordinary enhancement of strain hardening in fine-grained zirconium. Mater Sci Eng, A 591:167-172

3. Jiang L, Pérez-Prado MT, Gruber PA, Arzt E, Ruano OA, Kassner ME (2008) Texture, microstructure and mechanical properties of equiaxed ultrafine-grained $\mathrm{Zr}$ fabricated by accumulative roll bonding. Acta Mater 56(6):1228-1242

4. Toth LS, Gu C (2014) Ultrafine-grain metals by severe plastic deformation Mater. Mater Charact 92:1-14

5. Wang YL, Hui SX, Liu R, Ye WJ, Yu Y, Kayumov R (2014) Dynamic response and plastic deformation behavior of Ti-5Al-2.5Sn ELI and Ti-8Al-1Mo-1V alloys under high-strain rate. Rare Met 33:127-133. https://doi.org/10.1007/s12598-014-0238-y

6. Long Y, Wang T, Zhang HY, Huang XL (2014) Enhanced ductility in a bimodal ultrafinegrained Ti-6Al-4V alloy fabricated by high energy ball milling and spark plasma sintering. Mater Sci Eng, A 608:82-89

7. Berbenni S, Favier V, Berveiller M (2007) Impact of the grain size distribution on the yield stress of heterogeneous materials. Int J Plast 23:114-142. https://doi.org/10.1016/j.ijplas.2006. 03.004

8. Chong Y, Deng G, Gao S, Yi J, Shibata A, Tsuji N (2019) Yielding nature and Hall-Petch relationships in Ti-6Al-4V alloy with fully equiaxed and bimodal microstructures. Scripta Mater 172:77-82. https://doi.org/10.1016/j.scriptamat.2019.07.015

9. Pozdnyakov VA (2007) Ductility of nanocrystalline materials with a bimodal grain structure. Tech Phys Lett 33:1004-1006

10. Malygin GA (2008) Strength and plasticity of nanocrystalline metals with a bimodal grain structure. Phys Solid State 50:1032-1038

11. Ulacia I, Salisbury CP, Hurtado I, Worswick MJ (2001) Tensile characterization and constitutive modeling of AZ31B magnesium alloy sheet over wide range of strain rates and temperatures. J Mater Process Technol 211:830-839

12. Raeisinia B, Sinclair CW, Poole WJ, Tome CN (2008) On the impact of grain size distribution on the plastic behaviour of polycrystalline metals. Model Simul Mater Sci Eng 16(2):025001. https://doi.org/10.1088/0965-0393/16/2/025001

13. Revil-Baudard B, Cazacu O, Flater P, Chandola N, Alves JL (2016) Unusual plastic deformation and damage features in titanium: experimental tests and constitutive modeling. J Mech Phys Solids 88:100-122. https://doi.org/10.1016/j.jmps.2016.01.003

14. Aksyonov DA, Lipnitskii AG, Kolobov YuR (2013) Grain boundary segregation of C, N and O in hcp titanium from first-principles. Model Simul Mater Sci Eng 21:075009. https://doi.org/ 10.1088/0965-0393/21/7/075009

15. Skripnyak VA (2012) Mechanical behavior of nanostructured and ultrafine-grained materials under shock wave loadings. Experimental data and results of computer simulation. Shock compression of condensed matter. AIP Conf Proc 1426:965-970. https://doi.org/10.1063/1. 3686438

16. Skripnyak VA, Skripnyak EG (2017) Nanomechanics. Rijeka (Cratia): InTechOpen. (Article: Mechanical behaviour of nanostructured and ultrafine-grained metal alloy under intensive dynamic loading. Chapter 2: Nanotechnology and Nanomaterials). https://doi.org/10.5772/ intechopen.68291. Print ISBN 978-953+-51-3181-6

17. Skripnyak VA, Skripnyak NV, Skripnyak EG, Skripnyak VV (2017) Influence of grain size distribution on the mechanical behavior of light alloys in wide range of strain rates. AIP Conf Proc 1793:110001. https://doi.org/10.1063/1.4971664

18. Zhai J, Luo T, Gao X, Graham SM, Baral M, Korkolis YP, Knudsend E (2016) Modeling the ductile damage process in commercially pure titanium. Int J Solids Struct 91:26-45. https:// doi.org/10.1016/j.ijsolstr.2016.04.031

19. Zhao QY, Yang F, Torrens R, Bolzoni L (2019) In-situ observation of the tensile deformation and fracture behaviour of powder-consolidated and as-cast metastable beta titanium alloys. Mater Sci Eng, A 750:45-59. https://doi.org/10.1016/j.msea.2019.02.037

20. Zhu L, Shi S, Lu K, Lu J (2012) A statistical model for predicting the mechanical properties of nanostructured metals with bimodal grain size distribution. Acta Mater 60:5762-5772 
21. Zhu L, Lu J (2012) Modelling the plastic deformation of nanostructured metals with bimodal grain size distribution. Int J Plast 30-31:166-184

22. Clayton JD (2005) Dynamic plasticity and fracture in high density polycrystals: constitutive modeling and numerical simulation. J Mech Phys Solids 53:261-301

23. Magee AC, Ladani L (2015) Representation of a microstructure with bimodal grain size distribution through crystal plasticity and cohesive interface modeling. Mech Mater 82:1-12

24. Verleysen P, Peirs J (2017) Quasi-static and high strain rate fracture behaviour of Ti6Al4V. Int J Impact Eng 108:370-388. https://doi.org/10.1016/j.ijimpeng.2017.03.001

25. Armstrong RW, Zerilli FJ (1994) Dislocation mechanics aspects of plastic instability and shear banding. Mech Mater 17(2-3):319-327. https://doi.org/10.1016/0167-6636(94)90069-8

26. Döner M, Conrad H (1975) Deformation mechanisms in commercial Ti-5Al-2.5Sn (0.5 At. pct Oeq) alloy at intermediate and high temperatures (0.3-0.6Tm). Metall Trans A 6:853-861. https://doi.org/10.1007/BF02672308

27. Frost HJ, Ashby MF (1982) Deformation-mechanism maps. Pergamon Press, Oxford

28. Salem AA, Kalidindi SR, Doherty RD (2003) Strain hardening of titanium: role of deformation twinning. Acta Mater 51:4225-4237. https://doi.org/10.1016/s1359-6454(03)00239-8

29. Wang Q, Ren J, Wang Y, Xin C, Xiao L, Yang D (2019) Deformation and fracture mechanisms of gradient nanograined pure Ti produced by a surface rolling treatment. Mater Sci Eng, A 754:121-128. https://doi.org/10.1016/j.msea.2019.03.080

30. Herzig N, Meyer LW, Musch D, Halle T, Skripnyak VA, Skripnyak EG, Razorenov SV, Krüger L (2008) The mechanical behaviour of ultrafine grained titanium alloys at high strain rates. In: Proceedings of 3rd international conference on high speed forming-11-12 Mar 2008, Dortmund, Germany, pp 141-150. https://doi.org/10.17877/de290r-8660

31. Skripnyak VV, Kozulyn AA, Skripnyak VA (2019) The influence of stress triaxiality on ductility of $\alpha$ titanium alloy in a wide range of strain rates. Mater Phys Mech 42:415-422. https://doi. org/10.18720/MPM.4242019_6

32. Skripnyak VV, Skripnyak EG, Skripnyak VA (2020) Fracture of titanium alloys at high strain rates and under stress triaxiality. Metals 10(3):305. https://doi.org/10.3390/met10030305

33. Gao PF, Qin G, Wang XX, Li YX, Zhan M, Li GJ, Li JS (2018) Dependence of mechanical properties on the microstructural parameters of TA15 titanium alloy with tri-modal microstructure. Mater Sci Eng, A 739:203-213. https://doi.org/10.1016/j.msea.2018.10.030

34. Cayron C, Artaud B, Briottet L (2006) Reconstruction of parent grains from EBSD data. Mater Charact 57(4-5):386-401

35. Hémery S, Villechaise P (2019) In situ EBSD investigation of deformation processes and strain partitioning in bi-modal Ti-6Al-4V using lattice rotations. Acta Mater 171:261-274. https:// doi.org/10.1016/j.actamat.2019.04.033

36. Shi Y, Li M, Guo D, Ma T (2013) Tailoring grain size distribution for optimizing strength and ductility of multi-modal Zr. Mater Lett 108:228-230

37. Needleman A, Tvergaard V, Bouchaud E (2012) Prediction of ductile fracture surface roughness scaling. J Appl Mech 79(3):031015. https://doi.org/10.1115/1.4005959

38. Springmann M, Kuna M (2005) Identification of material parameters of the Gurson-TvergaardNeedleman model by combined experimental and numerical techniques. Comput Mater Sci 32:544-552. https://doi.org/10.1016/j.commatsci.2005.02.002

39. Tvergaard V (2015) Study of localization in a void-sheet under stress states near pure shear. Int J Solids Struct 60-61:28-34. https://doi.org/10.1016/j.ijsolstr.2015.08.008

40. Heibel S, Nester W, Clausmeyer T, Tekkayan AE (2017) Failure assessment in sheet metal forming using a phenomenological damage model and fracture criterion: experiments, parameter identification and validation. Procedia Eng 207:2066-2071. https://doi.org/10.1016/j.pro eng.2017.10.1065

41. Orozco-Caballero A, Li F, Esqué-de los Ojos D, Atkinson MD, Quinta da Fonseca J (2018) On the ductility of alpha titanium: the effect of temperature and deformation mode. Acta Mater 149:1-10. https://doi.org/10.1016/j.actamat.2018.02.022

42. Sharkeev YP, Vavilov VP, Belyavskaya OA, Skripnyak VA, Nesteruk DA, Kozulin AA, Kim VM (2016) Analyzing deformation and damage of VT1-0 titanium in different structural states 
by using infrared thermography. J Nondestr Eval 35(3):42. https://doi.org/10.1007/s10921016-0349-5

43. Yoo VH (1981) Slip, twinning, and fracture in hexagonal close-packed metals. Metall Trans A 12:409-418

44. Valoppi B, Bruschi S, Ghiotti A, Shivpur R (2017) Johnson-Cook based criterion incorporating stress triaxiality and deviatoric effect for predicting elevated temperature ductility of titanium alloy sheets. Int J Mech Sci 123:94-105. https://doi.org/10.1016/j.ijmecsci.2017.02.005

45. Zheng G, Tang B, Zhou Q, Mao X, Dang R (2020) Development of a flow localization band and texture in a forged near- $\alpha$ titanium alloy. Metals 10:121. https://doi.org/10.3390/met100 10121

46. Raabe D, Herbig M, Sandlöbes S, Li Y, Tytko D, Kuzmina M, Ponge D, Choi P-P (2014) Grain boundary segregation engineering in metallic alloys: a pathway to the design of interfaces. Curr Opin Solid State Mater Sci 18(4):253-261

47. Zhang B, Wang J, Wang Y, Wang Y, Li Z (2019) Strain-rate-dependent tensile response of Ti-5Al-2.5Sn alloy. Materials 12:659. https://doi.org/10.3390/ma12040659

48. Liang H, Pan FS, Chen YM, Yang JJ, Wang JF, Liu B (2011) Influence of the strain rates on tensile properties and fracture interfaces for $\mathrm{Mg}$ - $\mathrm{Al}$ alloys containing $\mathrm{Y}$. Adv Mater Res 284-286:1671-1677. https://doi.org/10.4028/www.scientific.net/amr.284-286.1671

49. Wei K, Zeng X, Huang G, Deng J, Liu M (2019) Selective laser melting of Ti-5Al-2.5Sn alloy with isotropic tensile properties: The combined effect of densification state, microstructural morphology, and crystallographic orientation characteristics. J Mater Process Technol 271:368-376. https://doi.org/10.1016/j.jmatprotec.2019.04.003

50. Yang L, Zhicong P, Ming L, Yonggang W, Di W, Changhui S, Shuxin L (2019) Investigation into the dynamic mechanical properties of selective laser melted Ti-6Al-4V alloy at high strain rate tensile loading. Mater Sci Eng, A 745:440-449. https://doi.org/10.1016/j.msea.2019.01.010

Open Access This chapter is licensed under the terms of the Creative Commons Attribution 4.0 International License (http://creativecommons.org/licenses/by/4.0/), which permits use, sharing, adaptation, distribution and reproduction in any medium or format, as long as you give appropriate credit to the original author(s) and the source, provide a link to the Creative Commons license and indicate if changes were made.

The images or other third party material in this chapter are included in the chapter's Creative Commons license, unless indicated otherwise in a credit line to the material. If material is not included in the chapter's Creative Commons license and your intended use is not permitted by statutory regulation or exceeds the permitted use, you will need to obtain permission directly from the copyright holder. 